\title{
Special discontinuities in models of continuum mechanics
}

\author{
Anna Chugaynova ${ }^{1, *}$ \\ ${ }^{1}$ Steklov Mathematical Institute of Russian Academy of Sciences, Moscow, Russia
}

\begin{abstract}
Some models of continuum mechanics with possible special discontinuities are analyzed. It is of interest to study such discontinuities since they can generate the non-uniqueness of solutions in a problem of arbitrary discontinuity disintegration.
\end{abstract}

\section{Introduction}

Special discontinuities, for example, deflagration waves, are known for a long time. These discontinuities are described as undercompressive shock wave $[1,2]$ and non-classical one [3] are used. We shall use the term a special discontinuity introduced in [4].

The solutions of nonlinear hyperbolic systems of equations are studied. To the hyperbolic system we add terms describing small-scale processes of dispersion and dissipation. Solutions of this supplemented system are smoothed discontinuous solutions of the initial system. The solution in the form of travelling wave for the supplemented system is called a stationary discontinuity structure. Among all discontinuities with stationary structures we select special discontinuities, their structures are described by integral curves joining two saddle points. The condition for such a curve to exist is called an additional condition on a discontinuity [4].This condition following from the requirement for a stationary structure to exist provides the evolutional character of a discontinuity [5].

If the initial system can have a special discontinuity as a solution, the problem of arbitrary discontinuity disintegration for this system can have non-unique solution [4, 6-9].

In what follows some continuum mechanics models for which special discontinuity formation is possible are listed and analyzed.

\section{Special discontinuities for longitudinal waves propagating in visco-elastic rods with complicated nonlinearity}

Special discontinuities in longitudinal waves propagating in visco-elastic rods were considered in [4, 6-8] (see also the references). Special discontinuities can occur if the function responsible for nonlinearity is specified in a special way. The discontinuity structure is described by the generalized KdV-Burgers equation

$$
\begin{gathered}
\frac{\partial v}{\partial t}+\frac{\partial \varphi(v)}{\partial x}=\mu \frac{\partial^{2} v}{\partial x^{2}}-m \frac{\partial^{3} v}{\partial x^{3}}, \\
m, \mu=\mathrm{const}, \quad v=v(x, t)
\end{gathered}
$$

*e-mail: anna_ch@mi.ras.ru 
The term involving the coefficient $m$ in the right-hand side of Eq. (1) describes the dispersion effects ( $m$ is the dispersion parameter). The term involving the coefficient $\mu$ takes into account the viscous effects and dissipation ( $\mu$ is the dissipation parameter). The nonlinearity is specified by the function $\varphi(v)$, which is not a quadratic one and will be defined below. One should analyze the traveling wave solutions representing shock structures in solutions of the equation

$$
\frac{\partial v}{\partial t}+\frac{\partial \varphi(v)}{\partial x}=0
$$

to which Eq.(1) is reduced when considering solutions characterized by variations of large scale $L$ in $x$ (both terms on the right-hand side of Eq. (1) become small as compared with the left-hand-side terms).

Equation (2) can be called a generalized Hopf equation, with the Hopf equation it coincides when $\varphi(v)$ is a quadratic function of $v$.

Equation (2) (as well as (1)) represents a conservation law, so the corresponding relation at the discontinuity can be written as

$$
W=\frac{[\varphi(v)]}{[v]} .
$$

Here, $W$ is the discontinuity velocity and square brackets denote the difference in the functions values behind and ahead of the discontinuity.

The function $\varphi(v)$ is defined as

$$
\varphi(v)=v^{4}-v^{2} .
$$

For $\mu \neq 0$, we make the following changes of variables in Eq. (1):

$$
t \rightarrow t \sqrt{m}, \quad x \rightarrow \mathrm{x} \sqrt{m}, \quad \gamma=\sqrt{m} / \mu, \quad \varphi(v)=f(u-1), \quad v=u-1 .
$$

Then Eq. (1) takes the form

$$
\frac{\partial u}{\partial t}+\frac{\partial f(u)}{\partial x}+\frac{\partial^{3} u}{\partial x^{3}}=\frac{1}{\gamma} \frac{\partial^{2} u}{\partial x^{2}}
$$

A stationary discontinuity structure is described by the equations

$$
\begin{gathered}
u=u(\xi), \quad \xi=x-W t, \\
\frac{d^{2} u}{d \xi^{2}}-\frac{1}{\gamma} \frac{d u}{d \xi}=W u-f(u), \\
\lim _{\xi \rightarrow-\infty} u(\xi)=u_{l}, \quad \lim _{\xi \rightarrow+\infty} u(\xi)=u_{r} .
\end{gathered}
$$

If problem (7) can be solved, then, from the point of view of a large length scale, its solution should represent a discontinuity with a stationary structure in which $u=u_{r}$ and $u=u_{l}$ are the states ahead of $(\xi>0)$ and behind $(\xi<0)$ the discontinuity. The states $u_{l}$ and $u_{r}$ satisfy relations (3) and, thus, represent the states behind and ahead of the discontinuity corresponding to the conservation law. In the plot $f(u)$ (see Figs. 1), the state ahead of the discontinuity (i.e., $u_{r}=0$ ) is denoted by the $A$.

The structure of the set of stationary-structured discontinuities is determined by dispersion, dissipation and a complex nonlinearity described by a nonmonotone potential of form (4). For $\varphi(u)$ thus defined, the solutions of Eq. (1) can involve special discontinuities. A discontinuity is called a special one if its structure represents a heteroclinic phase curve joining two saddle points in the $(u, d u / d \xi)$ - plane (one of these points is the state ahead of 


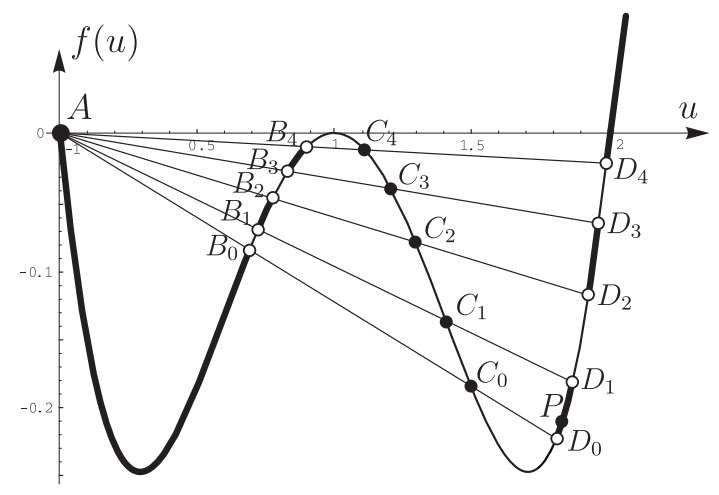

Figure 1. Set of discontinuities with a stationary structure for $\gamma \approx 22.8$.

the discontinuity while the other - the state behind the discontinuity). The number of special discontinuities grows with increasing $\gamma$; moreover, the entire set of stationary-structured discontinuities changes and becomes more complicated. This set consists of special and nonspecial discontinuities. The structure of a nonspecial discontinuity represents a phase curve joining two singular points, namely, the saddle (the state ahead of the discontinuity) and the focus or the node (the state behind the discontinuity).

When $\gamma$ is small, i.e., $0<\gamma<\gamma_{0}$ (with $\gamma_{0} \approx 1.19$ found in [8]), there are no special discontinuities. For weak dispersion, when $\gamma_{0}<\gamma<\gamma_{K_{1}}\left(\gamma_{K_{1}} \approx 10.54\right)$ found in [8]), the curve $f(u)$ has only one point $C_{0}$ corresponding to the state behind a special discontinuity.

Special discontinuities have a large effect on the pattern of the set of stationary-structured discontinuities. As $\gamma>\gamma_{K_{1}}$ grows, the number of special discontinuities also increases [4]. For sufficiently large $\gamma$, the set of states $u_{l}$ at given $u_{r}=0$ consists of segments and isolated points of the $\mathrm{u}$ axis. In Fig. 1, this set is shown by thick curve segments on the plot of $f(u)$ (curves $A B_{0}, B_{1} B_{2}, B_{3} B_{4}, D_{0} D_{1}, D_{2} D_{3}$ and the curve segment above the point $D_{4}$ ) and by the isolated points $C_{i}(i=0, \ldots, 4)$ corresponding to special discontinuities. It was obtained numerically in [8]) for the parameter values $u_{r}=0, m=1.3, \mu=0.05(\gamma \approx 22.8)$.

\section{Special discontinuities in nonlinearly-elastic media and dielectrics}

To approximately describe waves corresponding to characteristics with close velocities, we use the hyperbolic system of equations derived in [10]. This system describes nonlinear quasi-transverse waves in a weakly anisotropic elastic medium. Waves in which the particle displacements are approximately parallel to surfaces of constant phase are called quasitransverse ones. The system of equations has the form

$$
\begin{gathered}
\frac{\partial u_{\alpha}}{\partial t}+\frac{\partial}{\partial x}\left(\frac{\partial R\left(u_{1}, u_{2}\right)}{\partial u_{\alpha}}\right)=0, \quad \alpha=1,2 \\
R\left(u_{1}, u_{2}\right)=\frac{1}{2} f\left(u_{1}^{2}+u_{2}^{2}\right)+\frac{1}{2} g\left(u_{2}^{2}-u_{1}^{2}\right)-\frac{1}{4} \kappa\left(u_{1}^{2}+u_{2}^{2}\right)^{2}, \quad f, g, \kappa=\text { const }
\end{gathered}
$$

Here, $u_{\alpha}=u_{\alpha}(x, t)$ are the components of the shear strain of the medium, $u_{\alpha}=\partial w_{\alpha} / \partial x, w_{\alpha}$ is the displacement of a medium particle in the direction of the Cartesian axes $x_{\alpha}(\alpha=1,2)$ parallel to constant-phase planes, and $x$ is the Lagrangian coordinate of a medium particle. The constant $g$ is the anisotropy parameter, $\kappa$ is a constant characterizing nonlinear effects, 
and $f$ is the characteristic velocity (doubled) in the absence of nonlinearity and anisotropy (i.e., for $\kappa=0, g=0$ ). The sign of $\kappa$ has a large effect on the behavior of quasi-transverse simple and shock waves.

System (8) is associated with the jump conditions

$$
\left[\frac{\partial R}{\partial u_{\alpha}}\right]-W\left[u_{\alpha}\right]=0 .
$$

Similarly to the derivation of the Korteweg-de Vries-Burgers equation, Eqs. (8) are supplemented with terms describing dissipation and dispersion. The following equations were considered in [6]:

$$
\begin{aligned}
\frac{\partial u_{1}}{\partial t}+\frac{\partial}{\partial x}\left(\frac{\partial R\left(u_{1}, u_{2}\right)}{\partial u_{1}}\right) & =\mu \frac{\partial^{2} u_{1}}{\partial x^{2}}+m \frac{\partial^{2} u_{2}}{\partial x^{2}} \\
\frac{\partial u_{2}}{\partial t}+\frac{\partial}{\partial x}\left(\frac{\partial R\left(u_{1}, u_{2}\right)}{\partial u_{2}}\right) & =-m \frac{\partial^{2} u_{1}}{\partial x^{2}}+\mu \frac{\partial^{2} u_{2}}{\partial x^{2}}
\end{aligned}
$$

Here $\mu$ is the viscosity and $m$ is a coefficient responsible for dispersion. This system of equations was used to study the stationary structure of discontinuities in solutions to Eqs. (8).

Note that dispersion terms in Eqs.(8) can be represented in various ways. It was noted in [6] that the presence of dispersion terms with second derivatives in Eqs. (11) implies that the determining parameters of the medium include a pseudovector. If the latter is lacking, the dispersion terms of the lowest order of differentiation are third-order terms. In this case, the equations describing quasi-transverse waves in a weakly anisotropic elastic medium become

$$
\frac{\partial u_{\alpha}}{\partial t}+\frac{\partial}{\partial x}\left(\frac{\partial R\left(u_{1}, u_{2}\right)}{\partial u_{\alpha}}\right)=\mu \frac{\partial^{2} u_{\alpha}}{\partial x^{2}}-m \frac{\partial^{3} u_{\alpha}}{\partial x^{3}}, \quad \alpha=1,2
$$

System (12) describes nonlinear waves in nonlinearly elastic media and dielectrics [11].

For two different models (Eqs. (11) and (12)) the stationary structure problem is solved and a set of discontinuities with stationary structures is obtained. For each of these models the set of discontinuities with stationary structures involves special discontinuities. With dispersion growing the number of special discontinuities also grows.

\section{Conclusions}

Models of continuum mechanics are presented in which special discontinuities can occur. Solving the stationary structure problems allows to obtain the solutions representing special discontinuities. A set of such discontinuities depends essentially on small-scale processes inside the structure. If the viscosity is taken into account the number of special discontinuities grows with dispersion growing.

Special discontinuities can arise in other models of continuum mechanics which are described by known nonlinear wave equations (for example, the Sine-Gordon equation). The existence of special discontinuities ensures the existence of small-scale processes that determine the stationary structure of these discontinuities.

This work was supported by the Program of the Presidium of the Russian Academy of Sciences No 01 "Fundamental Mathematics and its Applications" under grant PRAS-18-01. 


\section{References}

[1] D. Jacobs, B. McKinney, M. Shearer, Journ. Diff. Equations 116, 448 (1995)

[2] G.A. El, M.A. Hoefer, M. Shearer, SIAM Review 59(1), 3 (2016)

[3] B. Hayes, M. Shearer, Proc. Roy. Soc. Edinburgh 129, 733 (1999)

[4] A.G. Kulikovskii, Soviet Phys. Dokl. 29, 283 (1984)

[5] P.D. Lax, Comm. Pure Appl. Math. 10, 537 (1957)

[6] A.G. Kulikovskii, A.P. Chugainova, Russ. Math. Surv. 63(2), 283 (2008)

[7] A.P. Chugainova, V.A. Shargatov, Comput. Math. Math. Phys. 56(2), 263 (2016)

[8] A.P. Chugainova, A.T. Il'ichev, A.G. Kulikovskii, V.A. Shargatov, J. Appl. Math. 82, 496 (2017)

[9] A.P. Chugainova, Comput. Math. Math. Phys. 57(6), 1013 (2017)

[10] A.G. Kulikovskii, J. Appl. Math. Mech. 50(4), 455 (1986)

[11] A.G. Kulikovskii, A.P. Chugainova, J. Exp. Theor. Phys. 110(5), 851 (2010) 